\title{
Huge fibroid uterus mimicking an ovarian malignancy
}

\author{
Sara Guleria*, Suman Thakur, Kushla Pathania
}

Department of Obstetrics and Gynecology, IGMC, Shimla, Himachal Pradesh, India

Received: 11 July 2021

Accepted: 06 August 2021

\author{
*Correspondence: \\ Dr. Sara Guleria, \\ E-mail: saraguleria94@gmail.com
}

Copyright: () the author(s), publisher and licensee Medip Academy. This is an open-access article distributed under the terms of the Creative Commons Attribution Non-Commercial License, which permits unrestricted non-commercial use, distribution, and reproduction in any medium, provided the original work is properly cited.

\begin{abstract}
Leiomyoma of the uterus is the most common benign tumor arising from uterine smooth muscle. This is a case of a woman with an exophytic uterine leiomyoma mimicking an ovarian neoplasm. The potential for uterine leiomyomas to grow to an extreme size before causing symptoms is quite remarkable. This is likely due to the relatively large volume of the abdominal cavity, the distensibility of the abdominal wall and the slow growth rate of these tumors. In our case report, despite a fibroid of size reaching up to 34 weeks of gestation, our patient had minimal symptoms except for abdominal discomfort. There were no symptoms related to abnormal uterine bleeding or abnormal bowel or bladder habit. Also, the history and clinical examination of the patient favored a diagnosis of ovarian malignancy.
\end{abstract}

Keywords: Ovarian malignancy, Fibroid, Subserosal

\section{INTRODUCTION}

Leiomyoma of the uterus is the most common benign tumor arising from uterine smooth muscle. The sizes of leiomyomas vary from being microscopic to being giant. Giant myomas are exceedingly rare. As leiomyomas enlarge, they outgrow their blood supply, which can lead to a cascade of inflammatory reactions and ischemic changes. ${ }^{1}$ This is a case of a woman with an exophytic uterine leiomyoma mimicking an ovarian neoplasm.

\section{CASE REPORT}

Mrs. Banti Devi w/o Tek Ram, 47-year-old female (P3+0) presented to the gynecology OPD of Kamla Nehru hospital, Shimla, with complaints of abdominal distension and discomfort for the past six months. She had normal menstrual cycles and no urinary or bowel complaints. She had no other significant past history.

On examination her general condition was fair with a blood pressure of $112 / 78 \mathrm{mmHg}$ in the right arm, in a sitting position with a pulse rate of 84 beats per min regular, respiratory rate of 16 breaths per min and a saturation of $96 \%$ on room air.
Also, there was no evidence of any lymphadenopathy.

On per abdomen examination: there was a large palpable non tender abdominopelvic mass reaching up to the xiphisternum. The mass was firm in consistency with a smooth surface and with restricted mobility. There was the presence of another mass in the hypogastrium with similar features. No shifting dullness was present.

On bimanual examination: The cervix was pushed towards the right side. The mass was tipped through all the fornices. The movement of the mass was not transmitted to the cervix.

Patient underwent routine preoperative blood investigations (complete hemogram, liver function tests, renal function tests, serum electrolytes and coagulation profile along with viral markers, random blood sugar and thyroid function tests) and tumour markers assessment (CA-125; CEA; CA19-9) which were all normal.

Ultrasonography was suggestive of an enlarged uterus with large multi lobulated fibroids with heterogeneous echotexture with increased vascularity (sarcomatous changes). 
On MRI: Large solid abdominopelvic mass lesion showing heterogeneous enhancement extending in the midline reaching up to the subhepatic region with nonvisualization of ovaries. This was giving a possibility of either large sub-serosal leiomyoma or ovarian origin tumour.

Patient was planned for an exploratory laparotomy. On opening the abdomen there was presence of around $100 \mathrm{cc}$ of peritoneal fluid which was sent for cytology. The uterus was enlarged to 34 weeks of gestation with normal bilateral ovaries and fallopian tubes. There was a large sub-serosal fibroid of about $15 \times 10 \mathrm{~cm}$ in size arising from the uterus and adherent to it.

Patient underwent a total abdominal hysterectomy with bilateral salpingo oophorectomy. The pelvic and paraaortic lymph nodes were not palpable. The mass along with the uterus weighed about $10 \mathrm{~kg}$. (Figure 1).

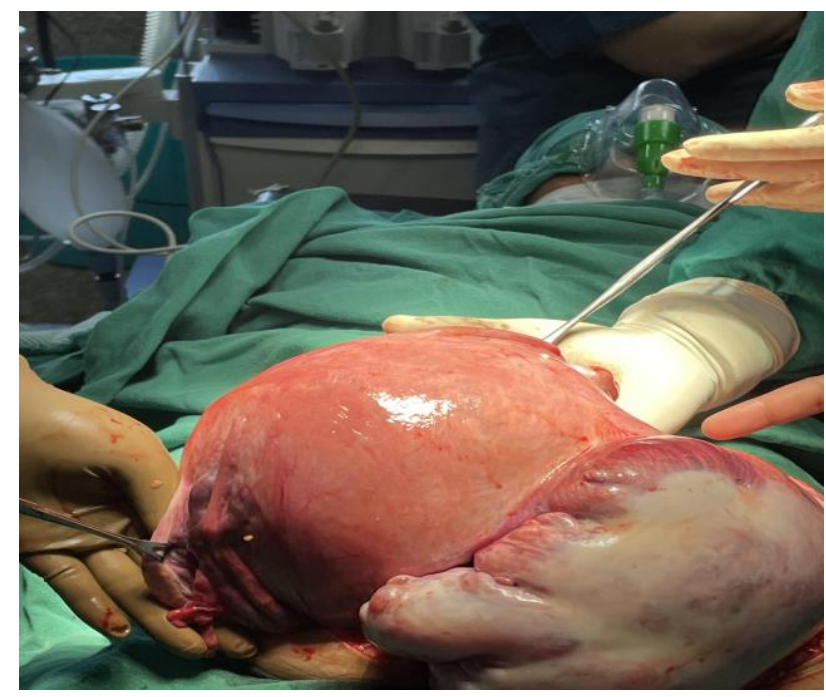

Figure 1: Uterus with fibroid viewed from in front.

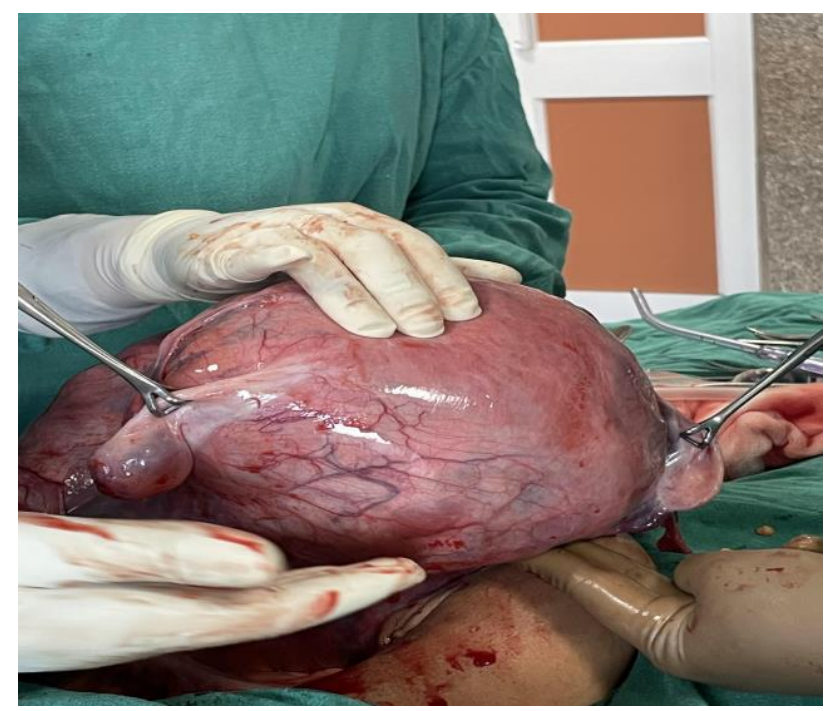

Figure 2: Uterus with bilateral ovaries.
On cut section: The uterocervical length was 6 inches.

The uterus had multiple intramural fibroids ranging from $1 \mathrm{~cm}$ to a maximum of $6 \mathrm{~cm}$ which had characteristic whorled appearance and no evidence of any degeneration or necrosis. There was also the presence of seedling fibroids.

The sub-serosal fibroid measured had a characteristic whorled appearance with no evidence of any degeneration or necrosis.

Postoperative period of the patient was uneventful and she was discharged on the $8^{\text {th }}$ post-operative day. Imprint cytology from the mass showed benign smears.

The histopathology report of the patient concluded multiple intramurals, submucosal and sub-serosal fibroids which showed histological features of leiomyoma with focal myxoid and hyaline degeneration.

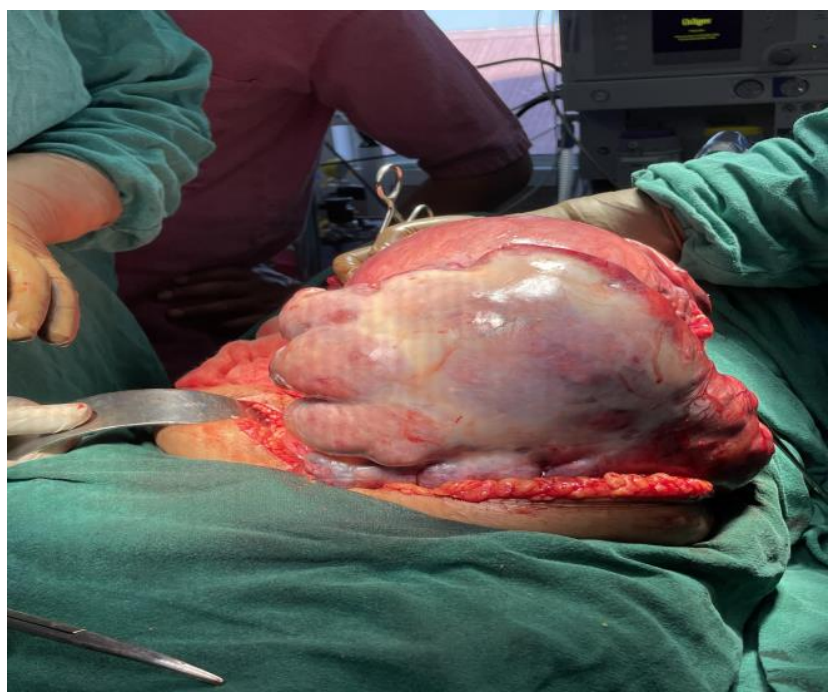

Figure 3: Subserosal fibroids.

\section{DISCUSSION}

Leiomyoma is the most frequently diagnosed gynecologic tumor, occurring in 20-30\% of women older than 30 years. It arises from the overgrowth of smooth muscle and connective tissue of the uterus. Histologically, a monoclonal proliferation of smooth muscle cells occurs. ${ }^{2}$

The potential for uterine leiomyomas to grow to an extreme size before causing symptoms is quite remarkable. This is likely due to the relatively large volume of the abdominal cavity, the distensibility of the abdominal wall and the slow growth rate of these tumors.

Submucosal and intramural fibroids typically manifest with abnormal uterine bleeding, whereas sub-serosal and pedunculated fibroids usually present with bulk-related symptoms of pelvic pain and bowel or bladder dysfunction. ${ }^{3}$ 
In our case, despite such a massive size of the fibroid the patients had no complaints in regard to the fibroids, their location and size. There were no menstrual complaints, urinary complaints, bowel dysfunction, pressure symptoms except for slight abdominal discomfort.

In literature, only a few cases of giant tumours have been reported, the largest uterine fibroid ever reported weighed $63.3 \mathrm{~kg}$ and was removed postmortem in $1888 .{ }^{4}$

Typical appearances of leiomyomas are easily recognized on imaging. However, the atypical appearances that follow degenerative changes can cause confusion in diagnosis such as adenomyosis, hematometra, uterine sarcoma and ovarian masses ${ }^{5}$

Rarely, uterine leiomyoma may undergo malignant degeneration to become a sarcoma. The incidence of malignant degeneration is less than $1.0 \%$ and has been estimated to be as low as $0.2 \% .^{6}$

The first differential diagnosis of a large mass with irregular septae and solid components filling the pelvic cavity is ovarian malignancy. This can be ruled out by visualizing normal ovaries or continuity of the mass with the uterus on imaging. Absence of ascites and elevated tumor markers may favor a diagnosis of a leiomyoma. ${ }^{7}$

The ovarian vascular pedicle sign noted on computed tomography (CT) is another way to confirm the ovarian origin of a pelvic mass and for differentiating the mass from a sub-serosal uterine fibroid. ${ }^{8}$

Yorita et al in a review article, noted that, among 17 cases mimicking ovarian tumors, most $(70 \%)$ were actually subserosal pedunculated masses and 6 cases had multilocular cystic morphology. ${ }^{9}$

Maizlin et al reported a case in which the sonographic features supported the diagnosis of an ovarian tumor however Magnetic Resonance Imaging aided the preoperative diagnosis of cystic degeneration of fibroids. ${ }^{10}$ Thus, MRI should be performed in all cases when there are huge cystic masses and the adnexa are not visualized.

\section{CONCLUSION}

In a case of a leiomyoma uterus severe symptoms may develop in 15 to $30 \%$ of cases, and the extent of symptoms depends on fibroid location, number, and size.

In our case report, despite a fibroid of size reaching up to 34 weeks of gestation, our patient had minimal symptoms except for abdominal discomfort. There were no symptoms related to abnormal uterine bleeding or abnormal bowel or bladder habit. Also, the history and clinical examination of the patient favoured a diagnosis of ovarian malignancy.
Therefore, any female presenting with large abdominopelvic mass should undergo a complete work up including tumour markers and an MRI scan. The differential diagnosis of a large fibroid mimicking an ovarian malignancy should always be kept in mind while evaluating such patients and preparing for surgery.

\section{Funding: No funding sources \\ Conflict of interest: None declared \\ Ethical approval: Not required}

\section{REFERENCES}

1. Rosai J. Rosai and Ackerman's Surgical Pathology: Female Reproductive System: Vulva, Vagina, Uterus, Fallopian tubes, Ovary, Placenta. $10^{\text {th }}$ ed. London: Mosby, 2011:1510.

2. Murase E, Siegelman ES, Outwater EK, Perez-Jaffe LA, Tureck RW. Uterine leiomyomas: histopathologic features, MR imaging findings, differential diagnosis, and treatment. Radiographics. 1999;19:1179-97.

3. Havryliuk Y, Setton R, Carlow JJ, Shaktman BD. Symptomatic fibroid management: systematic review of the literature. J Society Laparoendoscopic Surgeons. 2017;21(3).

4. Evans III AT, Pratt JH. A giant fibroid uterus. Obstet Gynecol 1979;54:385-6.

5. Aydin C, Eriş S, Yalçin Y, Selim HŞ. A giant cystic leiomyoma mimicking an ovarian malignancy. International journal of surgery case reports. $2013 \mathrm{Jan}$ $1 ; 4(11): 1010-2$.

6. Spanoudaki A, Oikonomou A, Dimitrova K. Pedunculated uterine leiomyoma mimicking abdominal mass: a case report. Cases J. 2008;1:315.

7. Degenerative Leiomyoma Mimicking an Ovarian Tumor: A Diagnostic Dilemma. J Gynecologic Surg. 2021;37(1):67-9.

8. Lee JH, Jeong YK, Park JK, Hwang JC. Ovarian vascular pedicle sign revealing organ of origin of a pelvic mass lesion on helical CT. Am J Roentgenol. 2003;181:131.

9. Yorita K, Tanaka Y, Hirano K, Kai Y, Arii K, Nakatani K, Ito S, Imai T, Fukunaga M, Kuroda N. A subserosal, pedunculated, multilocular uterine leiomyoma with ovarian tumor-like morphology and histological architecture of adenomatoid tumors: a case report and review of the literature. J med case rep. 2016;10(1):1-7.

10. Maizlin ZV, Vos PM, Cooperberg PL. Is It a fibroid? Are you sure? Sonography with MRI assistance. Ultrasound quarterly. 2007;23(1):55-62.

Cite this article as: Guleria S, Thakur S, Pathania K. Huge fibroid uterus mimicking an ovarian malignancy. Int J Reprod Contracept Obstet Gynecol 2021;10:3633-5. 